\title{
Activation of gab cluster transcription in Bacillus thuringiensis by $\gamma$-aminobutyric acid or succinic semialdehyde is mediated by the Sigma 54-dependent transcriptional activator GabR
}

\author{
Qi Peng ${ }^{1}$, Min Yang ${ }^{1}$, Wei Wang ${ }^{1,2}$, Lili Han ${ }^{1}$, Guannan Wang ${ }^{1,2}$, Pengyue Wang ${ }^{1,2}$, Jie Zhang ${ }^{1}$ and Fuping Song ${ }^{1 *}$
}

\begin{abstract}
Background: Bacillus thuringiensis GabR is a Sigma 54-dependent transcriptional activator containing three typical domains, an N-terminal regulatory domain Per-ARNT-Sim (PAS), a central $\mathrm{AAA}^{+}$(ATPases associated with different cellular activities) domain and a C-terminal helix-turn-helix (HTH) DNA binding domain. GabR positively regulates the expression of the gabT gene of the gab gene cluster, which is responsible for the $\gamma$-aminobutyric acid (GABA) shunt.

Results: Purified GabR was shown to specifically bind to a repeat region that mapped 58 bp upstream of the gabT start codon. The specific signal factors GABA and succinic semialdehyde (SSA) activated gabT expression, whereas GABA- and SSA-inducible gabT transcription was abolished in sigL and gabR mutants. GABA and SSA did not induce the expression of either SigL or GabR. Deletion of the PAS domain of GabR resulted in increased gabT transcriptional activity, both in the presence and absence of GABA.

Conclusions: This study identified the GabR-binding site on the gabT promoter; however, GabR does not bind to its own promoter. gabT transcription is induced by GABA and SSA, and inducible expression is dependent on SigL and activated by GabR. The PAS domain in GabR is repressing its enhancer transcriptional activity on the gabT promoter. Repression is released upon GABA addition, whereupon transcription is induced.
\end{abstract}

Keywords: GabR, Sigma 54, GABA, SSA, PAS domain

\section{Background}

The non-coded amino acid $\gamma$-aminobutyric acid (GABA) is found in a wide range of organisms, including bacteria, yeasts, plants and animals. In the adult brain, it is the predominant inhibitory neurotransmitter [1]. The GABA shunt, in which glutamate is converted to succinate, with GABA as an intermediate, is ubiquitous in most prokaryotic and eukaryotic organisms. Two important enzymes are involved in the GABA shunt: GABA transaminase (GABA-T, EC 2.6.1.19, encoded by $g a b T$ ), which catalyzes the reversible conversion of GABA to succinic semialdehyde (SSA), and succinic semialdehyde dehydrogenase

\footnotetext{
* Correspondence: fpsong@ippcaas.cn

${ }^{1}$ State Key Laboratory for Biology of Plant Diseases and Insect Pests, Institute of Plant Protection, Chinese Academy of Agricultural Sciences, Beijing, China Full list of author information is available at the end of the article
}

(SSADH, EC 1.2.1.16, encoded by $g a b D$ ), which irreversibly oxidizes SSA to succinate [2]. Both $g a b T$ and $g a b D$ have been identified in Escherichia coli [3], Rhizobium leguminosarum [4] and Bacillus subtilis [5].

Bacillus thuringiensis (Bt) has been widely commercialized throughout the world because of the production by strains of this species of insecticidal crystal proteins. During sporulation, Bt produces large crystalline parasporal inclusions. Previous studies suggested that the GABA shunt was related to the formation of Bt crystals and spores [6]. Bt possess a modified tricarboxylic acid cycle (TCA) in which $\alpha$-ketoglutarate dehydrogenase is absent. Instead, $\alpha$-ketoglutarate is converted to glutamate and then to succinate via $\gamma$-aminobutyric acid [7]. The GABA shunt allows circumvention of the $\alpha-$ ketoglutarate dehydrogenase step. Enzymatic analyses 
of Bt extracts suggested that a modified TCA operates during the sporulation phase of growth in conjunction with the glyoxylic acid cycle and the GABA pathway [6]. The Bt gab gene cluster is involved in GABA degradation. Previously, we proposed a model to explain the expression and regulation of the gab gene cluster in $\mathrm{Bt}$ strain HD73 [8]. In this model, three genes ( $g a b T$, $g a b R$ and $g a b D$ ) form two transcriptional units. $g a b T$ is separately transcribed, while $g a b R$ and $g a b D$ are cotranscribed. Moreover, we found that Sigma 54 RNA polymerase holoenzyme transcribes $g a b T$, with transcription activated by GabR.

Bacterial enhancer binding proteins (bEBPs) are transcriptional activators that assemble as hexameric rings in their active form, using ATP hydrolysis to modify the conformation of RNA polymerase containing the alternative sigma factor Sigma 54 [9]. bEBPs generally consist of three domains: the N-terminal regulatory domain plays a role in signal perception and modulates the activity of bEBP; the central $\mathrm{AAA}^{+}$domain is responsible for nucleotide binding, ATP hydrolysis, and oligomerization, and is sufficient to activate Sigma 54-dependent transcription; and the C-terminal DNA-binding domain contains a helix-turn-helix $(\mathrm{HTH})$ motif that enables enhancer site recognition by specific upstream activating sequences (UAS) [9]. The N-terminal or regulatory domains of many bEBPs respond to various environmental signals and thereby regulate the activity of the central $\mathrm{AAA}^{+}$domain [9]. For example, NtrC, DctD, ZraR and FlgR bEBPs are part of two-component systems that couple an external stimulus to an internal response [10-13]. These bEBPs have response regulator domains that are phosphorylated by specific sensor kinases. Certain other Sigma 54 activators have a regulatory domain that binds to small effector molecules, such as FhlA, which contains two GAF domains that bind formate to activate transcription of the formate hydrogen lyase system [14]. PAS (Per-ARNTSim) domains have been shown to bind a diverse array of ligands, including heme, flavins, 4-hydroxycinnamic acid, carboxylic acids, and divalent metal ions [15]. However, very few of the signals sensed by PAS domains in bEBPs have been identified. This is the case of TyrR bEBP-like protein, which in the presence of phenylalanine, tyrosine, or tryptophan, proposed to be sensed by the PAS domain, interact with the alpha-subunit of RNA polymerase to activate transcription [16].

GabR is a Sigma 54-dependent transcriptional activator that has three typical domains: an N-terminal PAS regulatory domain, a central $\mathrm{AAA}^{+}$domain, and a C-terminal HTH DNA-binding domain. In this study, we demonstrated that GabR binds upstream and in close proximity to the Sigma 54 binding site in the gabT promoter. Our data also show that the PAS domain in GabR is repressing its enhancer transcriptional activity on the gabT promoter.
Repression is released upon GABA addition, whereupon transcription is induced.

\section{Methods}

\section{Bacterial strains, plasmids, and growth condition}

The bacterial strains and plasmids used in this study are listed in Table 1. Bt strain HD73 was used throughout the study (accession numbers CP004069) [17]. E. coli strain TG1 was used as the host for cloning experiments. The Dam $^{-} / \mathrm{Dcm}^{-}$E. coli ET12567 strain (laboratory stock) was used to generate unmethylated DNA for the electrotransformation assay. Bt strains were transformed by electroporation, as described previously $[18,19]$. E. coli and $B t$ strains were cultured in Luria-Bertani (LB) medium, with $220 \mathrm{rpm}$ shaking, at 37 and $30 \mathrm{C}$, respectively. The antibiotic concentrations used for bacterial selection were as follows: $100 \mu \mathrm{g} / \mathrm{ml}$ kanamycin, $10 \mu \mathrm{g} / \mathrm{ml}$ erythromycin, and $10 \mu \mathrm{g} / \mathrm{ml}$ tetracycline for Bt, and $100 \mu \mathrm{g} / \mathrm{ml}$ ampicillin for $E$. coli. For $\beta$-galactosidase assays, bacteria were grown on Association of Official Analytical Chemists (AOAC) medium (Sigma, S5431, Saint-Quentin Fallavier, France) containing glucose $(7 \mathrm{mM})$ and Schaeffer s sporulation medium (SSM) [20]. Transcription from the Bt gabT promoter was induced by adding $0,1,2$, or $5 \mathrm{mM}$ of SSA and GABA to the AOAC culture medium.

\section{DNA manipulation techniques}

PCR was performed using Taq and KOD DNA polymerase (New England BioLabs Ltd., Beijing, China). Amplified fragments were purified using Axygen purification kits (Silicon Valley, CA, USA). Bt chromosomal DNA was extracted with the Puregene kit (Gentra, Minneapolis, MN, USA). Restriction enzymes and T4 DNA ligase (TaKaRa Biotechnology, Dalian, China) were used according to the manufacturers instructions. Oligonucleotide primers (Table 2) were synthesized by Sangon (Shanghai, China). E. coli plasmid DNA was extracted using the Axygen Plasmid Extraction Kit. All constructs were confirmed by DNA sequencing (BGI, Beijing, China).

\section{Total RNA isolation and 5'-RACE analysis}

For total RNA purification, strain HD73 was grown as previously described in SSM medium until the T7 stage of stationary phase (corresponding to $7 \mathrm{~h}$ after the end of the exponential phase) [21]. cDNA synthesis and transcriptional start sites (TSSs) of the gabT gene were determined using the SMARTer RACE cDNA Amplification Kit (Clontech, Mountain View, CA, USA) according to the manufacturers instructions. Gene-specific primers, gabTRACE, and the universal primer mix (UPM) (Table 2) were used to amplify the $5^{\prime}$ end of gabT mRNA. 
Table 1 Strains and plasmids used in this study

\begin{tabular}{|c|c|c|}
\hline Strain or plasmid & Relevant genotype and characteristics & Reference or source \\
\hline \multicolumn{3}{|l|}{ Strains } \\
\hline HD73 & Bt subsp. Kurstaki carrying the crylAc gene & Laboratory collection \\
\hline $\mathrm{HD}($ PgabT-lacZ) & Bt HD73 carrying pHT-PgabT plasmid; Em ${ }^{R}$ & {$[8]$} \\
\hline HD(PgabR-lacZ) & Bt HD73 carrying pHT-PgabR plasmid; Em ${ }^{R}$ & {$[8]$} \\
\hline HD(PgabTDR-lacZ) & Bt HD73 carrying pHT-PgabTDR plasmid; Em ${ }^{R}$ & This study \\
\hline HD(PgabTDI-lacZ) & Bt HD73 carrying pHT-PgabTDI plasmid; Em ${ }^{R}$ & This study \\
\hline HD(PsigL-lacZ) & Bt HD73 carrying pHT-PsigL plasmid; Em ${ }^{R}$ & This study \\
\hline$\Delta g a b T($ PgabT-lacZ) & $\mathrm{HD}\left(\Delta\right.$ gabT) carrying pHT-PgabT plasmid; $\mathrm{Em}^{\mathrm{R}}$ & {$[8]$} \\
\hline$\Delta g a b D(P g a b T-l a c Z)$ & $\mathrm{HD}(\Delta g a b D)$ carrying pHT-PgabT plasmid; Em ${ }^{R}$ & {$[8]$} \\
\hline$\Delta g a b R($ PgabT-lacZ) & $\mathrm{HD}(\Delta g a b R)$ carrying pHT-PgabT plasmid; Em ${ }^{R}$ & [8] \\
\hline$\Delta \operatorname{sig} L($ PgabT-lacZ) & $\mathrm{HD}\left(\Delta\right.$ sigL) carrying pHT-PgabT plasmid; $\mathrm{Em}^{\mathrm{R}}$ & {$[8]$} \\
\hline DPAS(PgabT) & $\mathrm{HD}(\Delta g a b R)$ carrying pHT-PgabT and pHT1618-DPAS plasmid; $\mathrm{Em}^{\mathrm{R}}$ and $\mathrm{Tet}^{\mathrm{R}}$ & This study \\
\hline CgabR(PgabT) & $\mathrm{HD}\left(\Delta\right.$ gabR) carrying pHT-PgabT and pHT1618-gabR plasmid; $\mathrm{Em}^{R}$ and $\operatorname{Tet}^{R}$ & This study \\
\hline E. coli TG1 & $\Delta\left(\right.$ lac-proAB) supE thi hsd-5 (F traD36 proA $A^{+}$pro $^{+}$lacla lacZ $\left.\Delta \mathrm{M} 15\right)$, general purpose cloning host & Laboratory collection \\
\hline E. coli ET12567 & $\begin{array}{l}F^{-} \text {dam-13::Tn9 dcm-6 hsdM hsdR recF143 zjj-202::Tn10 galK2 galT22 ara14 pacY1 } \\
\text { xyl-5 leuB6 thi-1, for generation of unmethylated DNA }\end{array}$ & Laboratory collection \\
\hline E.coli BL21(DE3) & E.coli $B, F^{-}, d c m$, ompT, hsdS(rB-mB-), gal, $\lambda(D E 3)$ & Laboratory collection \\
\hline BL21 (pET21b-gabR) & BL21(DE3) with pET21b-gabR plasmid & This study \\
\hline \multicolumn{3}{|l|}{ Plasmids } \\
\hline pET21b & Expressional vector, $A p^{R}, 5.4 \mathrm{~kb}$ & Laboratory collection \\
\hline pHT304-18Z & Promoterless lacZ vector, $\mathrm{Em}^{R}, \mathrm{Ap}^{R}$ & Laboratory collection \\
\hline pHT1618 & E. coli-Bt shuttle, Ap ${ }^{R}$, Tet ${ }^{R}$ & {$[25]$} \\
\hline pET21b-gabR & pET-21b containing gabR gene, $A^{R}$ & This study \\
\hline pHT-PgabT & pHT304-18Z carrying promoter upstream from gabT & {$[8]$} \\
\hline pHT-PgabR & pHT304-18Z carrying promoter upstream from gabR & {$[8]$} \\
\hline pHT-PgabTDR & pHT304-18Z carrying promoter upstream from gabT without GabR binding site & This study \\
\hline pHT-PgabTDI & $\begin{array}{l}\text { pHT304-18Z carrying promoter upstream from gabT without the first } 21 \text { repeat } \\
\text { region of GabR binding site }\end{array}$ & This study \\
\hline pHT-PsigL & pHT304-18Z carrying promoter upstream from sigL & This study \\
\hline pHT1618-gabR & pHT1618 containing gabR promter and gabR gene, $A p^{R}$ & This study \\
\hline pHT1618-DPAS & pHT1618 containing gabR promter and PAS domain deletion fragment, $A p^{R}$ & This study \\
\hline
\end{tabular}

\section{Expression and purification of GabR protein}

The expression plasmid pETgabR containing gabR from Bt strain HD73 was constructed by cloning the amplified gabR gene, using primers GabR-F (with a 5 BamHI restriction site) and GabR-R (with a 5 SalI restriction site), into pET21b previously digested with BamHI and SalI. pETgabR was transferred into E. coli BL21(DE3). Transformant $E$. coli cells were grown to exponential phase in LB medium supplemented with ampicillin at 37C. The expression of GabR-His protein was induced with $1 \mathrm{mM}$ (final concentration) isopropyl- $\beta$-D-thiogalactopyranoside for $3 \mathrm{~h}$ at $37 \mathrm{C}$. Cells were harvested by centrifugation at $13,500 \mathrm{~g}$ for $10 \mathrm{~min}$ and suspended in $10 \mathrm{mM}$ imidazole NPB buffer (10 mM imidazole, $1 \mathrm{M} \mathrm{NaCl}, 20 \mathrm{mM}$ sodium phosphate, $\mathrm{pH}$ 7.4). Bacteria were lysed on ice by sonication using an ultrasonic cell disruption system (Mini-Beadbeater-96, Biospec). The bacterial lysate was centrifuged at 16,000 $\mathrm{g}$ for $10 \mathrm{~min}$ at $4 \mathrm{C}$, and supernatant containing solubilized GabR-His protein was recovered, filtered through a $0.45-\mu \mathrm{m}$ membrane filter, and loaded onto a HiTrap chelating column (1 ml, Pharmacia). After binding the protein, the column was washed with $10 \mathrm{mM}$ imidazole NPB solution and the target GabR-His protein was eluted with NPB solution containing a stepwise gradient of imidazole from 100 to $500 \mathrm{mM}$.

\section{Gel mobility shift assays}

The PgabT DNA fragment was obtained by PCR of strain HD73 genomic DNA using primers PgabT-F and PgabT-R, which were previously labeled according to a fluorescent 
Table 2 Primers used in this study

\begin{tabular}{|c|c|}
\hline oligonucleotides & sequence $(5-3)^{a}$ \\
\hline PgabT-F & GATTGCTATGCAATTGGGGTGC \\
\hline PgabT-R & CCTITCTITACATTGTTTTCTC \\
\hline gabTRACE & CGAGCGATCTTCACCGCGTTCTCAACT \\
\hline UPM & AAGCAGTGGTATCAACGCAGAGTACGCGGG \\
\hline GabR-F & CGGGATCCGATGGTTGCAGAAAAGGAACG \\
\hline GabR-R & GCGTCGACTCCAATGTTTTCTTCCTCCTCTATG \\
\hline PgabTDR-F & AACTGCAGGAACAAGCCTTGATGTAGCGAA \\
\hline PgabTDR-1 & CTATAAAGACTCTTAAAAAGAAAGTTGGCA \\
\hline PgabTDR-2 & TGCCAACTTICTIITAAAGAGTCTITATAG \\
\hline PgabTDR-R & CGGGATCCCCTITTCTTTACATTGTTTCTC \\
\hline PgabTDI-1 & GTCTAATGTGTAAGG TTAATGAGTCTTAT \\
\hline PgabTDI-2 & ATAAAGACTCATTAACCTTACACATTAGAC \\
\hline PsigL-F & CCCAAGCTTATAGAGCGGTGGTTTCCGGTAC \\
\hline PsigL-R & CGGGATCCAATAATCTCCCCCTTGTTTCTATTG \\
\hline PgabRD-F & AACTGCAGGTGCTGGTGTACCGATAAG \\
\hline PgabRD-R & CGGGATCCCGATCCACTTCACTACCTT \\
\hline CgabR-1 & CGGGATCCGTGCTGGTGTACCGATAAGT \\
\hline CgabR-2 & CCGGAATTCTTATCCAATGTTTTCTTCCTCC \\
\hline DPAS-F & GGGATCGTTGTGCGTAATCAGCTAAAAAC \\
\hline DPAS-R & GTITTAGCTGATTACGCACAACGATCC \\
\hline
\end{tabular}

Restriction enzyme sites are italics.

5'-end 6-FAM modification and confirmed by DNA sequencing. Electrophoresis mobility shift assays (EMSA) were performed as previously described [22] to analyze the binding of purified GabR protein to PgabT DNA. Briefly, the PgabT DNA probe $(0.1 \mu \mathrm{g})$ was incubated with different concentrations of purified GabR at $25 \mathrm{C}$ for $20 \mathrm{~min}$ in binding buffer $(10 \mathrm{mM}$ Tris $\mathrm{HCl}, 0.5 \mathrm{mM}$ dithiothreitol (DTT), $50 \mathrm{mM} \mathrm{NaCl}, 500 \mathrm{ng}$ poly (dI:dC), $\mathrm{pH} 7.5$ and $4 \%$ $(\mathrm{v} / \mathrm{v})$ glycerol) in a total volume of $20 \mu \mathrm{l}$. The DNA-protein mixtures were applied to non-denaturing $5 \%(\mathrm{w} / \mathrm{v})$ polyacrylamide gels in TBE buffer $(90 \mathrm{mM}$ Tris-base, $90 \mathrm{mM}$ boric acid, 2 mM EDTA, pH 8.0) for resolution of the complexes, using a Mini-PROTEAN system (Bio-Rad) at $160 \mathrm{~V}$ for $1 \mathrm{~h}$. Signals were visualized directly from the gel with the FLA Imager FLA-5100 (Fujifilm). The specificity of the shift was confirmed using poly ( $\mathrm{dI}: \mathrm{dC}$ ), and GST protein, bovine serum albumin (BSA), and as negative controls, the cry $1 A c$ and gerE promoters (which do not bind to GabR protein; data not shown). Increasing concentrations (0.1$50 \mu \mathrm{M})$ of SSA or GABA were incubated with the PgabT probe and GabR to analyze the effect of SSA and GABA on the binding of GabR and PgabT.

\section{DNase I footprinting assays}

DNase I footprinting assays were performed based on a fluorescent labeling procedure [23]. Briefly, gabT promoter DNA was PCR-amplified using the fluorescently-labeled primers PgabT-F and PgabT-R (Table 2) and purified from an agarose gel. The labeled PgabT DNA probe (120 ng) was incubated for $20 \mathrm{~min}$ at $25 \mathrm{C}$ with the indicated concentrations of purified GabR and BSA in a total volume of $50 \mu \mathrm{l}$ of binding buffer (described above for EMSA). DNase I digestion was then carried out for $1 \mathrm{~min}$ at $25 \mathrm{C}$ and stopped with stop buffer (Promega). After phenolchloroform extraction and ethanol precipitation, the samples were loaded on an Applied Biosystems 3730 DNA genetic analyzer together with an internal-lane size standard (ROX-500, Applied Biosystems). A dye primer-based sequencing kit (Thermo) was used to precisely determine the sequences after their alignment wtih the capillary electrophoresis results from the reactions. Electropherograms were analyzed with GeneMarker v1.8 (Applied Biosystems).

\section{Construction of the PgabT-lacZ fusion bearing a deletion of GabR-binding site}

A fragment containing the gabT promoter with the GabRbinding site deleted was cloned in fusion with the lac $Z$ gene. The construct was obtained as follows: The downstream and upstream regions of the gabT promoter were amplified in two PCR reactions, using strain HD73 DNA as the template, and PgabTDR-F/PgabTDR-2 and PgabTDR-1/PgabTDR-R primers, respectively. These DNA fragments were fused by overlapping PCR using the primer pair PgabTDR-F/PgabTDR-R. The resulting PCR products were digested with PstI and BamHI, purified, and ligated into the low-copy-number vector pHT304-18Z [24], which harbors a promoterless $l a c Z$ gene. The recombinant plasmid named pHT-PgabTDR, which has a deletion of $60 \mathrm{bp}$ corresponding to the GabR-binding site, was introduced into Bt strain HD73, yielding the transformant strain HD (PgabTDR-lacZ). Another recombinant plasmid, pHTPgabTDI, which has a deletion of the first 21-bp repeat located in the GabR-binding site region, was constructed by a similar method. The downstream and upstream regions of the gabT promoter were amplified in two PCR reactions, using strain HD73 DNA as the template, and PgabTDR-F/PgabTDI-2 and PgabTDI-1/PgabTDI-R primers, respectively. These DNA fragments were fused by overlapping PCR using the primer pair PgabTDR-F/PgabTDR-R. The PCR products were digested with PstI and BamHI, purified, and ligated into the vector pHT304-18Z. The resulting plasmid, pHT-PgabTDI, was introduced into Bt strain HD73 yielding the transformant strain HD (PgabTDI-lacZ).

\section{Construction of sigL and gabR promoters with lacZ gene fusion}

In strain HD73, Sigma 54 is encoded by the sigL gene. $313 \mathrm{bp}$ fragment from the sigL promoter was amplified from strain HD73 DNA using primers PsigL-F (with 5' - 
HindIII) and PsigL-R (with 5'-BamHI) (Table 2). The HindIII-BamHI restriction fragment of the sigL promoter was cloned into the vector pHT304-18Z. The recombinant plasmid, pHT-PsigL, was introduced into Bt strain HD73 yielding the transformant strain HD (PsigL-lacZ).

A fragment containing $633 \mathrm{bp}$ of the $g a b R$ promoter was previously cloned [8]. Here, a truncated $g a b R$ promoter fragment of $580 \mathrm{bp}$ was constructed, deleting the $-12 /-24$ conserved element. The 580-bp gabR promoter fragment was amplified from strain HD73 DNA using PgabRD-F (with 5'-PstI) and PgabRD-R (with 5'-BamHI) primers (Table 2). The PstI-BamHI fragment of gabR promoter was then ligated into the vector pHT304-18Z [24]. The recombinant plasmid pHT-PgabRD was introduced into Bt strain HD73, and the resulting strain was named HD (PgabRD-lacZ).

\section{Complementation of the gabR mutant and deletion of PAS domain}

A DNA fragment containing $g a b R$ and the $g a b R$ promoter was amplified with CgabR-1 and CgabR-2 primers (Table 2) using Bt strain HD73 DNA as template. The PCR product $(1,974 \mathrm{bp})$ was digested with $\mathrm{BamHI}$ and EcoRI and ligated into plasmid pHT1618 [25]. The resulting plasmid (pHT1618-gabR) was amplified in E. coli and introduced into the Bt mutant strain $\triangle g a b R$ (PgabT-lacZ). This plasmid complements the $g a b R$ mutant strain and allows evaluation of the expression of the $g a b T$ promoterlacZ fusion.

A similar plasmid carrying the $g a b R$ promoter and $g a b R$ gene but without the PAS domain encoded sequence, was constructed by amplifiying two DNA fragments using strain HD73 DNA as the template and CgabR-1/DPAS-R and DPAS-F/CgabR-2 primers, respectively (Table 2 ). The amplified fragments were fused by an overlapping PCR using CgabR-1 and CgabR-2 primers. The final PCR product $(1,689 \mathrm{bp})$ was digested with EcoRI and BamHI, purified and ligated into plasmid pHT1618. The resulting plasmid (pHT1618-DPAS) was amplified in E. coli and introduced into the Bt mutant strain $\triangle g a b R$ (PgabT-lacZ). This plasmid complements the gabR strain, with a $g a b R$ gene where the PAS domain (amino acids 33128 of GabR) was deleted, and allows evaluation of the gabT promoterlacZ fusion in $\beta$-galactoside activity assays. The resulting strains CgabR(PgabT) and DPAS(PgabT) were selected based on their erythromycin and tetracycline resistance and identified by PCR.

\section{$\beta$-galactosidase assays}

Bt strains containing lac $Z$ transcriptional fusions were cultured in AOAC medium (supplemented with $7 \mathrm{mM}$ glucose) at $30 \mathrm{C}$ and $220 \mathrm{rpm}$. GABA or SSA at concentrations of $0,0.2,1$ and $5 \mathrm{mM}$ was added to the cultures when the $\mathrm{OD}_{600}$ reached 1.0 (mid-exponential phase, defined as $\mathrm{A}_{0}$ ). For PgabT and PgabR activity assay without GABA or SSA, $\mathrm{T}_{0}$ is the end of the exponential phase $\left(\mathrm{OD}_{600}=2.0-2.2\right)$, and $\mathrm{Tn}$ is $\mathrm{n}$ hours after the end of the exponential phase. Samples of $2.0 \mathrm{ml}$ were taken at 1-h intervals after induction. Cells were harvested by centrifugation for $1 \mathrm{~min}$ at $12,000 \mathrm{~g}$, and the supernatant was discarded. The $\beta$-galactosidase-specific activities were determined as previously described [26] and expressed as Miller units. The values reported represent averages from at least three independent assays.

\section{GATTGCTATGCAATTGGGGTGCTATCTGGTACGGGAGACCGTCCAACA}

TTAGAACCACTTGCTGATTTAGTGTTAGATTCTGTTGGAGAGTTTATT

TCTCAATCGGGTGAGTTTTTCTGGGAGAAAGAAAAGTCTAATGTGTAA

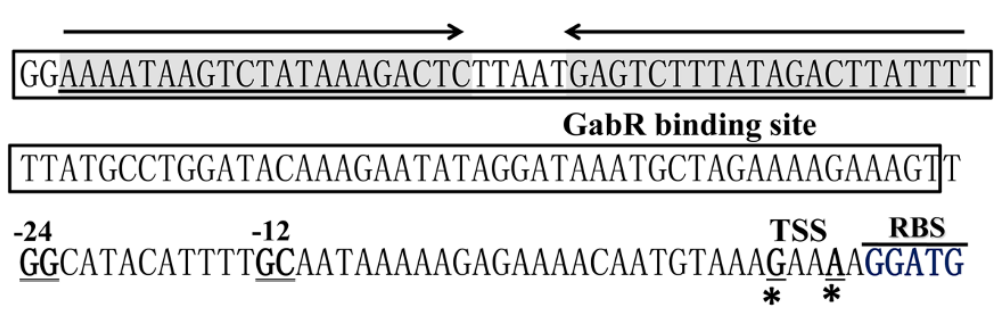

\section{GGGTACGCAATGAACACAAAA}

GabT

Figure 1 Nucleotide sequence of the gabT upstream region. Transcriptional start sites (TSSs, indicated by asterisks) are located 16 and 19 bp upstream from the start codon of the gabT gene (underlined). The putative ribosome-binding-site (RBS) is indicated by an upper line. The $-12,-24$ sequences are double underlined. The GabR binding site (boxed region) maps 58 bp upstream of the start codon of gabT. A 21-bp repeat region (underlined, gray and arrow) maps 105 bp upstream of the gabT start codon. 
B

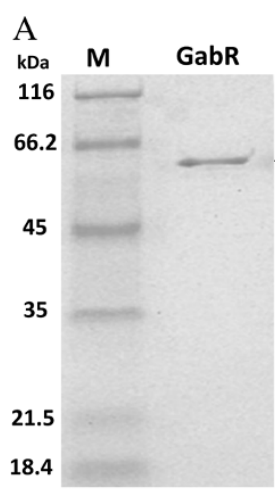

$\stackrel{57 \mathrm{kDa}}{ }$
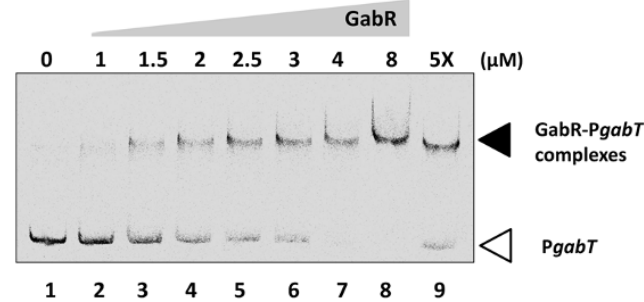

$\mathrm{C}$

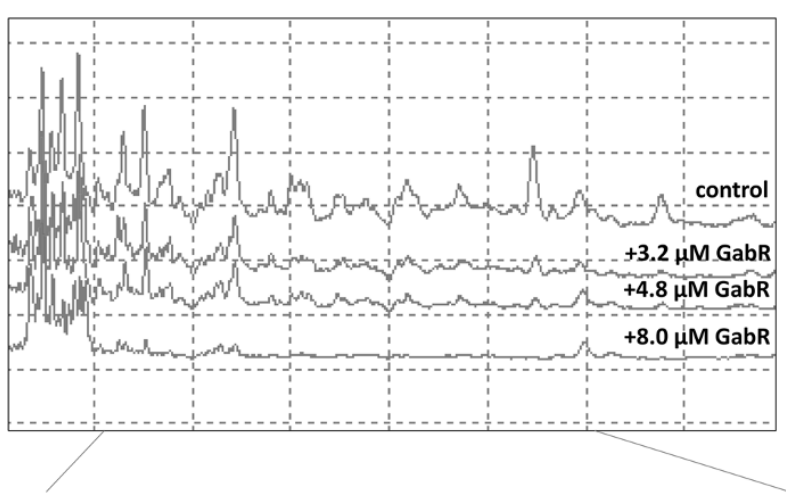

5' - GGAAAATAAGTCTATAAAGACTCTTAATGAGTCTTTATAGACTTATTT

TTTATGCCTGGATACAAAGAATATAGGATAAATGCTAGAAAAGAAAGT -3'

$\mathrm{D}$

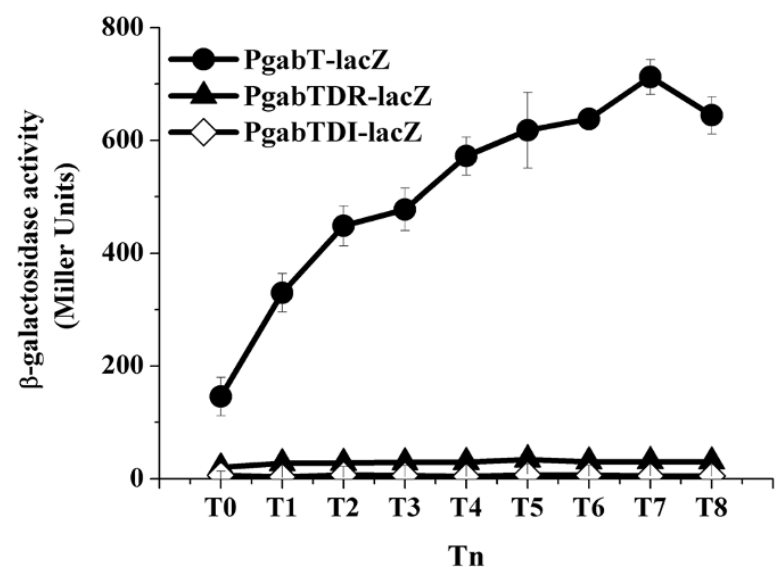

Figure 2 Identification of the GabR-binding site in the gabT promoter. Panel A, SDS-PAGE analysis of GabR (57 kDa) expressed in E. coli (pET21b-gabR) and purified by nickel affinity column chromatography. M, protein marker. Panel B, Electrophoresis mobility shift assay (EMSA) of the gabT promoter fragment (286 bp) after interaction with GabR. Lane 1, FAM-labeled PgabT probe; lanes 2 8, incubation of the probe with increasing concentrations of purified GabR indicated at the top of the figure. Each lane contained $0.1 \mu \mathrm{g}$ of probe. Lane 9, incubation of a five-fold greater amount of unlabeled PgabT probe mixed with labeled PgabT probe and $8 \mu \mathrm{M}$ GabR. Panel C, protection of a 96-bp sequence in the gabT promoter by GabR, as revealed in a DNase I footprinting protection assay. The fluorograms correspond to the control DNA (10 $\mu \mathrm{M}$ BSA) and to the protection reactions (with 3.2, 4.8 and $8 \mu \mathrm{M}$ of GabR). Panel D, $\beta$-galactosidase activity assay of the gabT promoter with the GabR-binding site $(\bullet)$, without the 60-bp GabR-binding site $(\mathbf{\Lambda})$, and without the first 21-bp repeat region of the GabR-binding site ( () ). The deletion sequence is that underlined in Figure $2 C . T_{0}$ is the end of exponential phase, and $T n$ is $n$ hours after $T_{0}$. 


\section{Results}

Determination of the transcriptional start site of $g a b T$ To determine the transcription start site (TSS) of gabT, 5 '-RACE analysis was performed as described in the Materials and Methods. According to the sequences of 16 random clones, a $\mathrm{G}$ located 19 bp upstream from the start codon was identified in eight, and an A residue located 16 bp upstream of the start codon in the remaining eight. Thus two TSSs were located 16 and 19 bp upstream of the ATG start codon of gabT (Figure 1). The typical ribosome binding site (RBS) sequence (-AGGATG-) was identified at an appropriate distance upstream from the start codon of gabT. The sequence TGGCATACATTT GCA was identified upstream of the $g a b T$ start codon and was similar to the $-12 /-24$ consensus sequence (BYGG CMYRNNNYYGCW) of Sigma 54-binding sites [27].

However, the two TSSs were located $25 \mathrm{bp}$ and $28 \mathrm{bp}$ from the putative -12 promoter element. In several Sigma 54-dependent promoters, the initiation of transcription is not precisely located 12 nt downstream from the conserved GC element [28]. For instance, in the case of the Rhodospirillum centenum cheAY promoter, the TSS is located 8 nt downstream of the -12 element [29]. In the Pseudomonas aeruginosa algD promoter, the TSS and -12 elements are $21 \mathrm{nt}$ apart [30].

\section{Identification of a GabR-binding site in the gabT promoter} fragment

To determine whether GabR is able to bind the $g a b T$ promoter, GabR-His protein was expressed in E. coli and purified to near-homogeneity using $\mathrm{Ni}^{2+}$-affinity chromatography. The mobility of GabR-His in SDS-PAGE corresponded well to its predicted molecular mass of $57 \mathrm{kDa}$ (Figure 2A). The ability of GabR to bind to a DNA fragment containing the PgabT (286 bp) promoter was examined by EMSA. FAM-labeled fragments containing the promoter regions of $g a b T$ were incubated with different amounts of GabR and assayed for the formation of protein-DNA complexes. Slower-migrating probe-protein complexes were observed upon incubation with increasing amounts of GabR (Figure 2B). A five-fold molar excess of unlabeled PgabT probe competed with the labeled PgabT probe, indicating specific binding (Figure 2B, lane 9). GabR did not bind to other labeled promoters from strain HD73, such as cry1Ac and gerE (data not shown). These results indicate that GabR recognizes and specifically binds to sequences within the $g a b T$ promoter fragment.

To precisely determine the GabR-binding site in the $g a b T$ promoter, DNase I footprinting assays were carried out using the same $g a b T$ promoter fragment used in the EMSA. A 96-bp fragment was protected by GabR binding (Figure 2C) (corresponding to the boxed sequence in the gabT region shown in Figure 1). Moreover, a 21-bp repeat region mapped $105 \mathrm{bp}$ upstream of the start codon of gabT (Figure 1), with the 21-pb inverted repeat separated by 5 bp (shaded gray in Figure 1).

To determine whether the proposed 96-bp sequence is the GabR binding site in vivo, a 60-bp fragment of the binding site was deleted from the gabT promoter and the promoter carrying the deletion was fused to $l a c Z$ (PgabTDR-lacZ). The deleted sequence is underlined in Figure 2C. Another deletion fragment, in which the first 21-bp repeat located at the GabR-binding site region was removed, was similarly fused to lacZ (PgabTDI-lacZ). The deleted sequence is double-underlined in Figure $2 \mathrm{C}$. The $\beta$-galactosidase assay showed that the activities of HD(PgabTDR-lacZ) and HD(PgabTDI-lacZ) strains were sharply decreased compared with that of wild-type strain HD73 carrying the gabT promoter fused to lacZ (PgabTlacZ) (Figure 2D). This result shows that disruption of the proposed GabR-binding site prevents GabT expression in vivo.
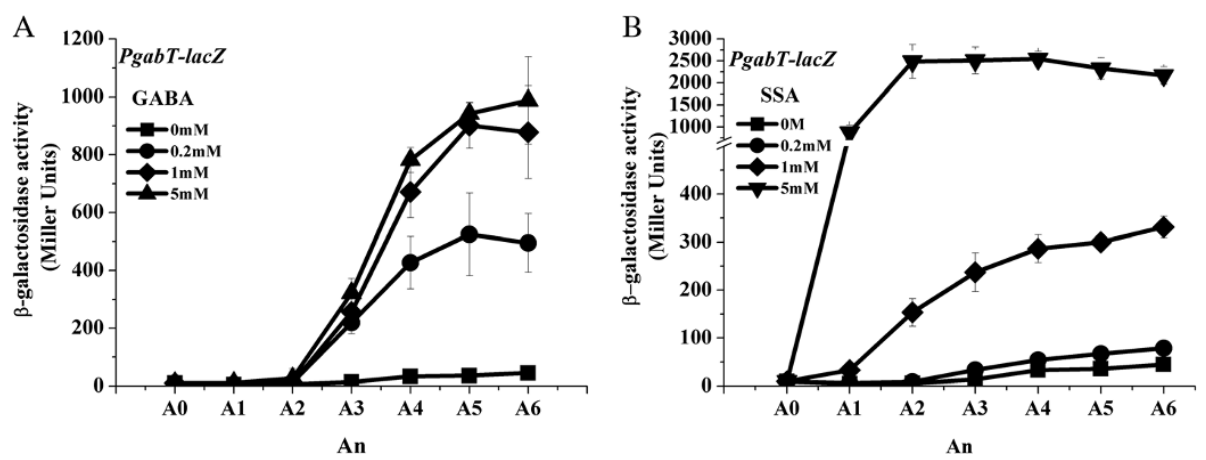

Figure 3 Induction of $g a b T$ transcription by GABA and SSA. Bt strains containing a PgabT-lacZ transcriptional fusion were cultured in AOAC medium. At $\mathrm{OD}_{600}=1.0\left(\mathrm{~A}_{0}\right)$, GABA and SSA were added at different concentrations. Samples were collected at 1-h intervals for $6 \mathrm{~h}$ after induction and $\beta$-galactosidase activity was assayed. Panel A, GABA was added to strain HD(PgabT-lacZ) at a final concentration of $0 \mathrm{mM}(\mathbf{\bullet}), 0.2 \mathrm{mM}(\bullet)$, $1 \mathrm{mM}(\bullet)$ and $5 \mathrm{mM}(\boldsymbol{\Delta})$. Panel B, SSA was added to strain HD(PgabT-lacZ) at a final concentration of $0 \mathrm{mM}(\boldsymbol{\bullet}), 0.2 \mathrm{mM}(\bullet), 1 \mathrm{mM}(\bullet)$ and $5 \mathrm{mM}(\boldsymbol{\nabla})$. $A_{0}$ is mid-exponential phase $\left(\mathrm{OD}_{600}=1.0\right)$, and $A n$ is $n$ hours after $A_{0}$. 


\section{GABA and SSA induce gabT expression}

The PgabT-lacZ fusion showed lower activity in AOAC medium (Figure 3) than in SSM (Figure 2D). To analyze the inducible activity of the gabT promoter, cells were cultured in AOAC medium. The addition of 0.2, 1 and $5 \mathrm{mM}$ GABA or SSA induced the expression of a PgabTlacZ fusion (Figure 3A and B), confirming the induction of gabT expression by these two signaling molecules. However, they differed in their gabT transcription induction patterns: GABA caused induction after $\mathrm{A}_{3}$ (3 hours after the mid-exponential phase), whereas SSA caused nearly immediate induction. These results might be explained if we hypothesize that SSA is the direct signal, while GABA is the precursor of SSA signal synthesis.

Induction of gabT promoter activity by GABA and SSA depends on Sigma 54 and GabR

In a previous study, we demonstrated that $g a b T$ expression is controlled by Sigma 54, via the transcription activator GabR [8]. Here, we analyzed the effect of $1 \mathrm{mM}$ GABA or SSA on PgabT transcription activity in sigL (Sigma 54 coding gene in $\mathrm{Bt}$ ) or gabR mutants. Neither GABA nor SSA induced activation of the gabT promoter in $\triangle s i g L$ or $\triangle g a b R$ (Figure $4 \mathrm{~A}$ and B). These data showed that GabR and Sigma 54 are absolutely necessary for gabT

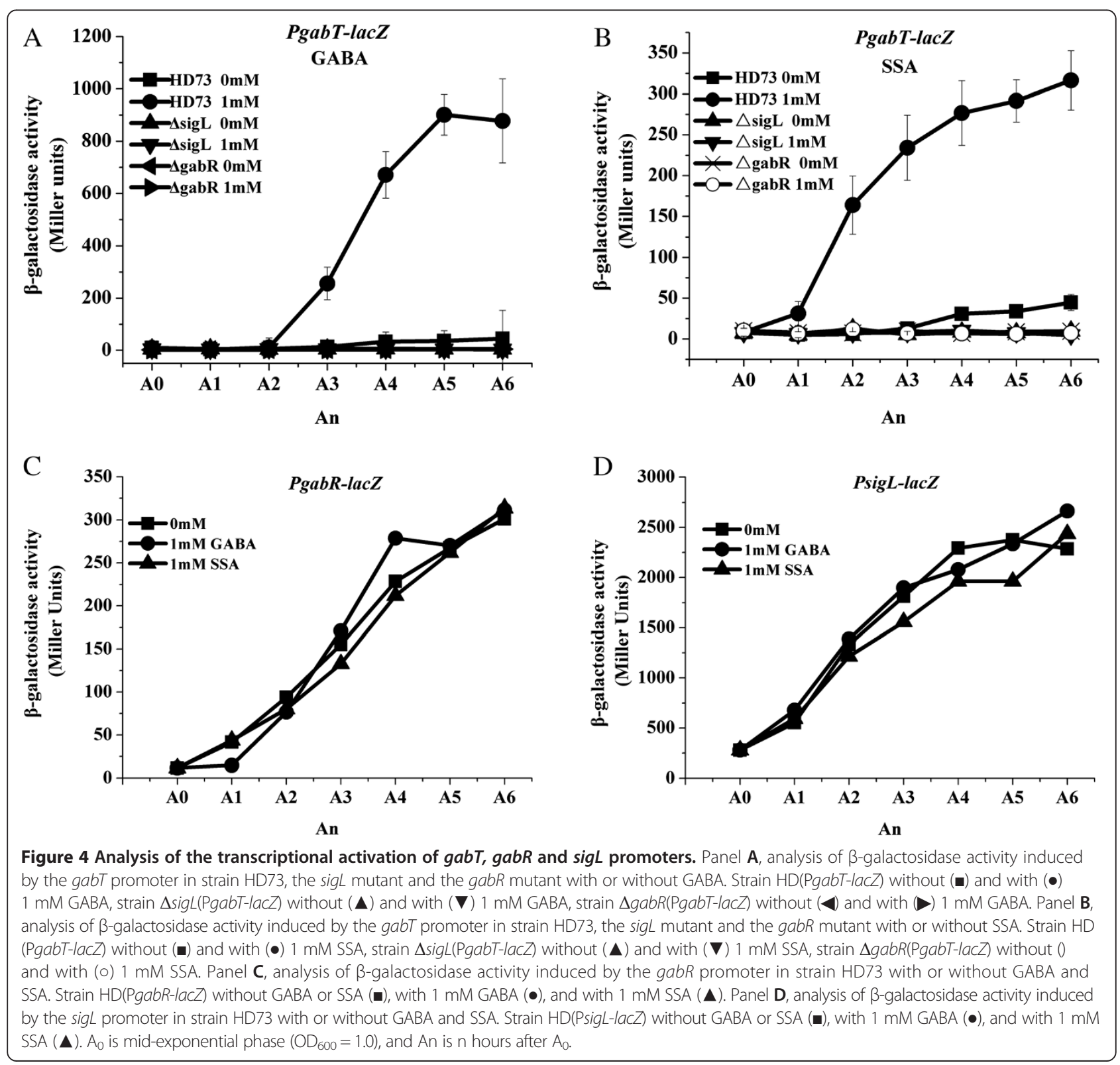


transcription and that GABA- or SSA-induced gabT transcription is controlled by Sigma 54 and activated by GabR bEBP.

PgabT induction is not caused by GABA or SSA enhancement of the transcriptional activity of gabR or sigL To determine whether GABA and SSA induced transcription of the gabT promoter by affecting sigL and gabR transcription, strains carrying sigL and $g a b R$ promoter fusions with lacZ were used $[\mathrm{HD}(\mathrm{P}$ sigL-lacZ) and $\mathrm{HD}(\mathrm{P} g a b R-$ lacZ)]. The addition of GABA and SSA had no effect on either PgabR or PsigL transcriptional activities (Figure 4C and D), suggesting that the GABA- and SSA-induced transcription from the $g a b T$ promoter is not due to the increased expression of sigL or gabR. Increasing concentrations of GABA and SSA did not enhance the binding of GabR to the gabT promoter (Additional file 1: Figure S1). Thus, the induction of PgabT by GABA and SSA is not caused by activating GabR and DNA binding.
The $g a b R$ promoter is not a Sigma 54-dependent promoter In the following, we provide additional evidence that transcription from the gabR promoter is not dependent on SigL, nor is it activated by GabR, which differs from the conclusion reached in our previous study [8]. EMSA performed to analyze the binding of GabR to the PgabR promoter showed that GabR did not bind to its own gabR promoter (Figure $5 \mathrm{~B}$ ). The $\beta$-galactosidase activity of the $g a b R$ promoter was tested again in the sigL and gabR mutants. The results failed to showed significant difference in the $g a b R$ promoter activities of wild-type strain HD73 and the sigL and gabR mutants (Figure 5C). Furthermore, deletion of a Sigma 54-like -12/-24 conserved region in the gabR promoter (PgabRD highlighted in Figure 5A) had no effect on PgabR transcription (Figure 5D). It should be noted that several reports have shown that deletion of one or more nucleotides in the stretch included in the $-24 /-12$ elements of Sigma 54dependent promoters abolishes promoter function [31,32].
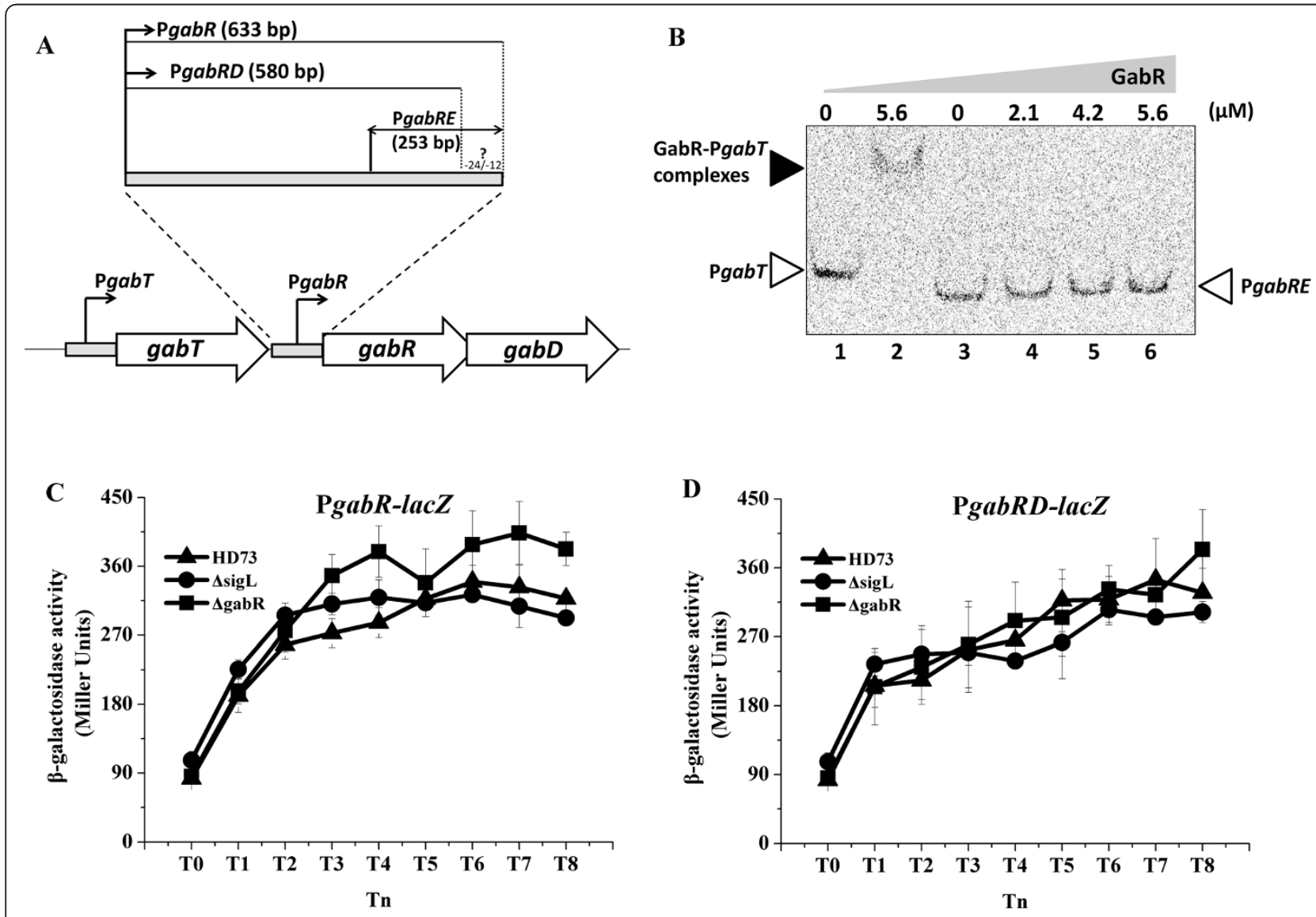

Figure 5 Analysis of the role of Sigma 54 and GabR on gabR transcription. Panel A, Model of the gabR upstream region. The PgabR (633 bp) fragment contains the wild-type promoter, the PgabRD(580 bp) fragment of the gabR promoter without the -12/-24-like elements, and the PgabRE (253 bp) fragment is used for GabR binding. Panel B, EMSA for PgabRE after interaction with GabR. Lane 1, FAM-labeled PgabT probe; Lane 2, probe PgabT was incubated with $5.6 \mu \mathrm{M}$ of purified GabR. Lane 3 6, the FAM-labeled PgabRE probe was incubated with increasing concentrations of purified GabR as shown at the top of the figure. Each lane contained $0.1 \mu \mathrm{g}$ of probe. C. $\beta$-galactosidase activity assay of PgabR in wild-type strain HD73 ( $\mathbf{\Lambda}$ ),

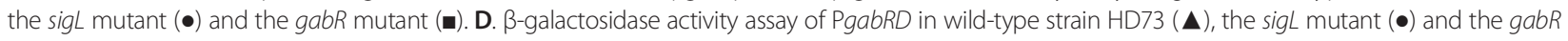
mutant ( $\mathbf{\square}) . T_{0}$ is the end of exponential phase, and $T n$ is $n$ hours after $T_{0}$. 
Those studies proposed that there is a stringent requirement for these motifs to be positioned on the same face of the DNA helix as a necessary condition for the binding of RNA polymerase and Sigma 54 [28]. Since there are only $11 \mathrm{nt}$ in $-24 /-12$ elements of the gabR promoter, it is not a typical Sigma 54-dependent promoter. Furthermore, GABA and SSA had no effect on the binding of GabR to the gabT promoter, as judged by EMSA assays (data not shown), suggesting that GABA and SSA activation of PgabT is not caused by enhanced GabR-binding to DNA.

\section{The PAS domain of GabR plays a role in the GABA-inducible transcription of $g a b T$}

GabR is a Sigma 54-dependent transcriptional activator containing three typical domains, similar to those in other bEBPs, including a PAS domain in the $\mathrm{N}$-terminal region (Figure 6A). To determine the role of PAS domain in GABA-induced transcription of gabT, a PAS domain deletion in GabR was constructed and its capacity to induce the gabT promoter was compared with that of wild-type GabR. The GabR $\triangle$ PAS mutant (DPAS) and GabR wildtype (CgabR) proteins were then used to complement a GabR mutant. The transcriptional activity of gabT was determined by measuring $\beta$-galactosidase activity of the fusion protein (see Methods). As shown in Figure 6B, gabT promoter activity in strain DPAS(PgabT) was higher than that of strain $\mathrm{CgabR}(\mathrm{P} g a b T)$ without the addition of GABA. Interestingly, GABA had no effect on the transcriptional activity of DPAS (PgabT) (Figure 6B). These data suggest that the PAS domain in GabR is repressing its enhancer transcriptional activity on the gabT promoter. Repression is released upon GABA addition, whereupon transcription is induced.

\section{Discussion}

The structure of the $g a b$ gene cluster in Bt is different from those found in E. coli and B. subtilis [8]. We confirmed that expression of $g a b T$ in Bt is positively regulated by GabR, which is a Sigma 54-dependent transcriptional activator, and that GabR specifically binds to the gabT promoter. Transcriptional activation of gabT by GabR is mediated by the signal molecules GABA and SSA. In Bt, GabR is not involved in gabR transcriptional activation and GABA and SSA had no effect on sigL or gabR transcription. By contrast, in B. subtilis, gabD and gabT form a GABAinducible operon, with GabR, encoded by a divergent gene, $g a b R$, acting as a transcriptional regulator of both the gabTD operon and its own gene [33]. In B. subtilis, the presence of both GABA and pyridoxal 5 '-phosphate (PLP) is required for GabR-dependent activation of the gabT promoter. The efficiency of the GabR-dependent repression of the $g a b R$ transcript is not affected by the addition of PLP or GABA [33]. In this work we showed that the

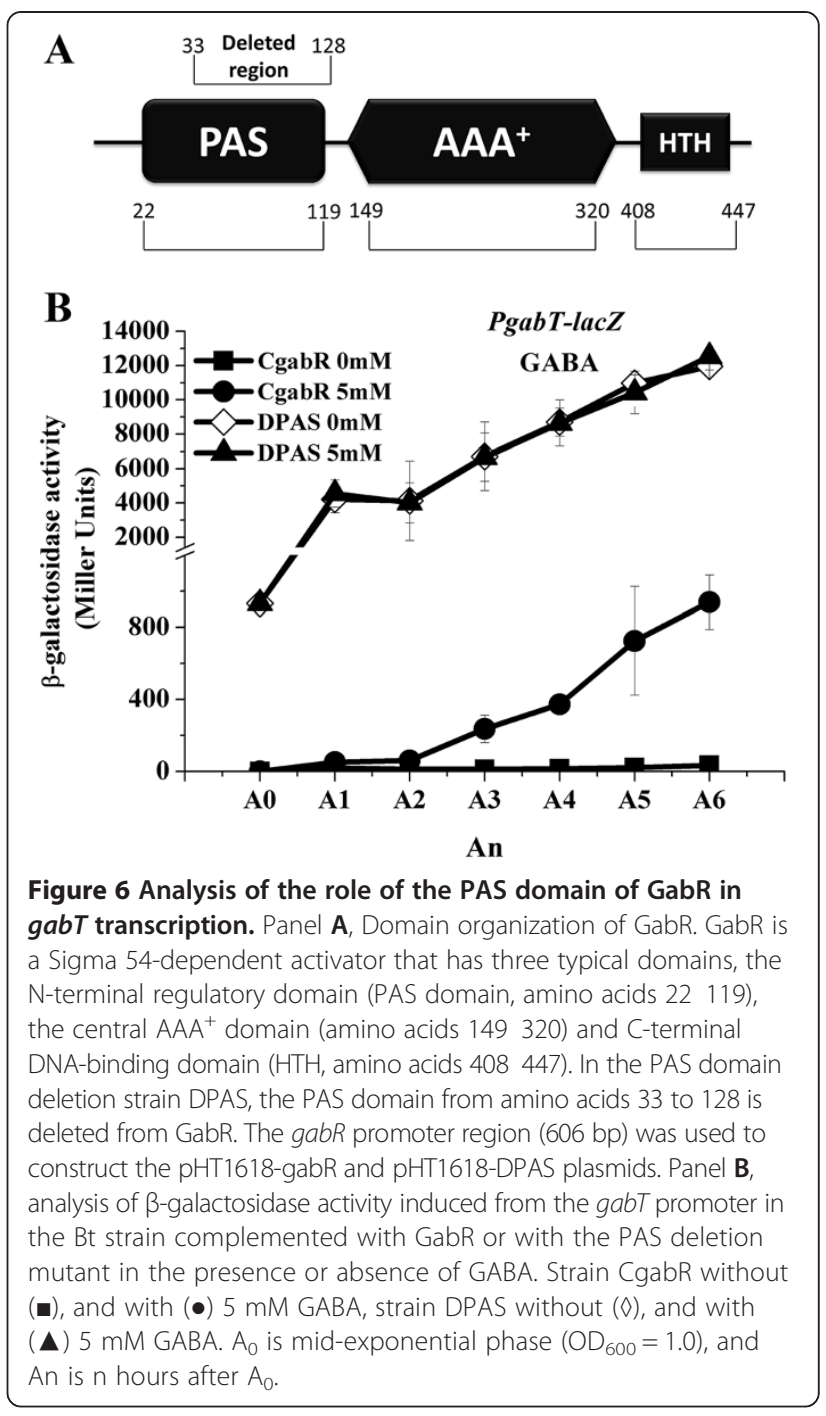

GABA-inducible mechanism in Bt is different from that in B. subtilis.

These different induction mechanisms can be attributed to the different GabR structures found in Bt and B. subtilis. GabR of B. subtilis is a member of the understudied MocR/GabR subfamily of the GntR family of transcription regulators [34]. GabR in B. subtilis has a short Nterminal HTH-containing domain and a long C-terminal domain that is similar to full-length aminotransferases or to the PLP-binding domain of aminotransferases [33]. The GabR structure provides a model for DNA binding at the gabR-gabT locus by one or more GabR homodimers. GabR aminotransferase-like activity involving GABA and PLP is not essential to its primary function as a transcriptional regulator [34]. However, GabR in Bt is a Sigma 54-dependent transcriptional activator, also referred to as a bacterial enhancer binding protein (bEBP). GabR comprises three domains (Figure 6A), including an N-terminal PAS domain. Here, we showed that the PAS domain of 


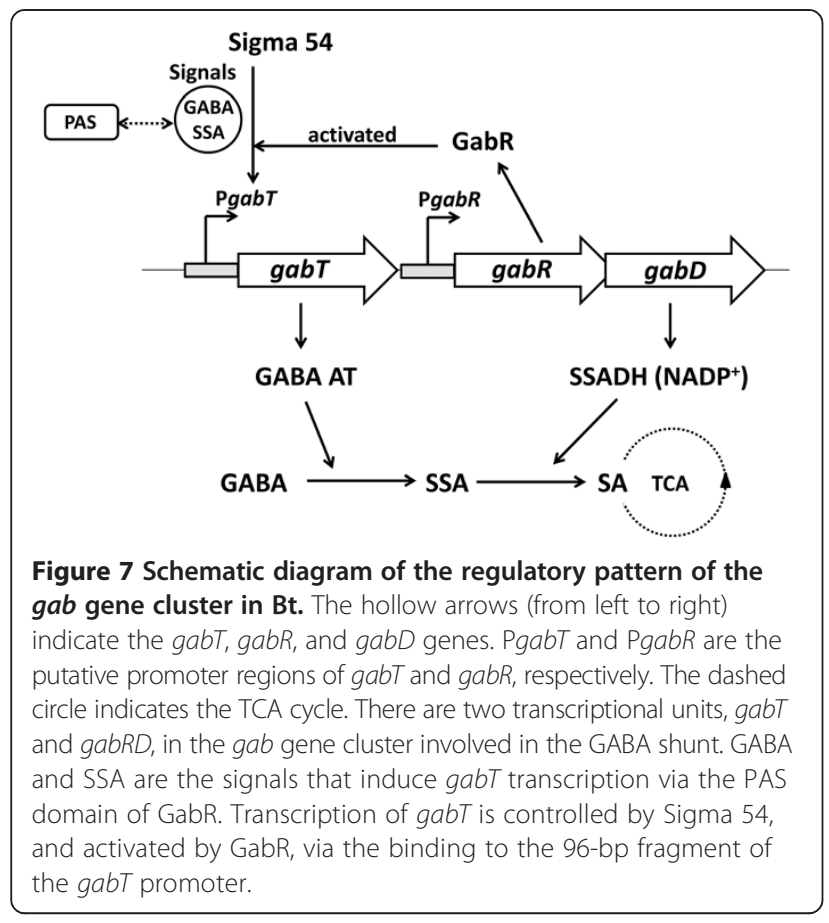

GabR, which is the signal sensor domain, is required for the regulation of GABA-inducible transcriptional activity of the $g a b T$ promoter.

The role of the N-terminal regulatory domain of bEBPs has been documented in many Sigma 54-dependent transcriptional activators, whereas little is known about the function of the PAS domain, even though PAS domain is present in many bEBPs. Indeed, only the PAS domain of the bEBP-like protein TyrR has been investigated [16]. TyrR contains both a PAS domain and an ACT domain and it facilitates the activation or repression of the transcription of genes involved in aromatic amino acid biosynthesis and transport. In this study, we found that deletion of the PAS domain of GabR in Bt results in induced gabT expression and that induction is not dependent on GABA. The gabT promoter activity in the PAS domain deletion strain was higher than that in the strain containing wild-type GabR. These data suggests that the PAS domain of GabR acts as a signal sensor domain that is repressing GabR enhancer transcriptional activity on the gabT promoter. Repression is released upon GABA addition, whereupon transcription is induced. This model agrees with the proposed function of the $\mathrm{N}$-terminal regulatory domain of bEBPs in modulating the activity of bEBPs. PAS domains have been shown to bind a diverse array of ligands [15]. In the case of $\mathrm{Bt}$, it is proposed that the PAS domain of GabR binds to GABA and/or SSA. It will be interesting to determine whether the PAS domain of other bEBPs functions in a similar way, i.e., by repressing bEBPs enhancer transcriptional activity after the sensing of signal ligands.

\section{Conclusions}

The data presented here improve our understanding of the regulation of the gab gene cluster in Bt and form the basis of a new regulatory model is proposed herein (Figure 7). Two transcriptional units, gabT and $g a b R D$, in the $g a b$ gene cluster are involved in the GABA shunt. GABA and SSA are the signals involved in $g a b T$ transcription, via the PAS domain of GabR. Transcription of gabT is controlled by Sigma 54, and activated by GabR, through binding to a $96 \mathrm{bp}$ region on the $g a b T$ promoter. The PAS domain of GabR acts as a signal sensor domain, modulating GabR activity. The data suggest that the PAS domain in GabR is repressing its enhancer transcriptional activity on the $g a b T$ promoter. Repression is released upon GABA addition, whereupon transcription is induced.

\section{Additional file}

Additional file 1: Figure S1. EMSA for GabR-PgabT with different concentration of SSA (A) or GABA (B). Lane 1, FAM-labeled PgabT probe; Lane 2-6, Incubation of PgabT probe and $2.5 \mu \mathrm{M}$ GabR with the increasing concentrationsof SSA or GABA indicated at the top of the figure. Each lane contained $0.1 \mu \mathrm{g}$ of probe.

\section{Competing interests}

The authors declare that they have no competing interests.

\section{Authors contributions}

QP and FS designed the research. QP performed the experimental work and drafted the manuscript. MY and LH purified the protein and performed the EMSA. WW performed the 5 RACE analysis and inducible activity. GW and PW constructed the PAS domain deletion strain and carried out $\beta$-galactosidase assay. FS and JZ critically revised the manuscript for intellectual content. All authors read and approved the final version of the manuscript.

\section{Acknowledgements}

This work was supported by grant from the National Natural Science Foundation of China (Nos. 31270111 and 31300085) and the National High Technology Research and Development Program of China (863 Program; 2011AA10A203). We thank Dr. Mario Soberon and Dr. Alejandra Bravo from Instituto de Biotecnologia, Universidad Nacional Autonoma de Mexico, and Dr. Didier Lereclus from the Institut National de la Recherche Agronomique for their critical suggestions.

\section{Author details}

${ }^{1}$ State Key Laboratory for Biology of Plant Diseases and Insect Pests, Institute of Plant Protection, Chinese Academy of Agricultural Sciences, Beijing, China. ${ }^{2}$ College of Life Sciences, Northeast Agriculture University, Harbin, China.

Received: 30 June 2014 Accepted: 24 November 2014

Published online: 20 December 2014

\section{References}

1. Varju P, Katarova Z, Madarasz E, Szabo G: GABA signalling during development: new data and old questions. Cell Tissue Res 2001, 305(2):239 246.

2. Shelp BJ, Bown AW, McLean MD: Metabolism and functions of gamma-aminobutyric acid. Trends Plant Sci 1999, 4(11):446 452.

3. Bartsch K, von Johnn-Marteville A, Schulz A: Molecular analysis of two genes of the Escherichia coli gab cluster: nucleotide sequence of the glutamate:succinic semialdehyde transaminase gene ( $g a b T)$ and characterization of the succinic semialdehyde dehydrogenase gene (gabD). J Bacteriol 1990, 172(12):7035 7042. 
4. Prell J, Bourdes A, Karunakaran R, Lopez-Gomez M, Poole P: Pathway of gamma-aminobutyrate metabolism in Rhizobium leguminosarum 3841 and its role in symbiosis. J Bacterio/ 2009, 191(7):2177 2186.

5. Belitsky BR, Sonenshein AL: GabR, a member of a novel protein family, regulates the utilization of gamma-aminobutyrate in Bacillus subtilis. Mol Microbiol 2002, 45(2):569 583

6. Aronson JN, Borris DP, Doerner JF, Akers E: Gamma-aminobutyric acid pathway and modified tricarboxylic acid cycle activity during growth and sporulation of Bacillus thuringiensis. Appl Microbiol 1975, 30(3):489 492.

7. Nickerson KW, De Pinto J, Bulla LA Jr: Sporulation of Bacillus thuringiensis without concurrent derepression of the tricarboxylic acid cycle. J Bacteriol 1974, 117(1):321 323

8. Z Zhu L, Peng Q, Song F, Jiang Y, Sun C, Zhang J, Huang D: Structure and regulation of the gab gene cluster, involved in the gamma-aminobutyric acid shunt, are controlled by a sigma54 factor in Bacillus thuringiensis. J Bacteriol 2010, 192(1):346 355.

9. Bush M, Dixon $\mathrm{R}$ : The role of bacterial enhancer binding proteins as specialized activators of sigma54-dependent transcription. Microbiol Mol Biol Rev 2012, 76(3):497 529.

10. Reitzer L: Nitrogen assimilation and global regulation in Escherichia coli. Annu Rev Microbiol 2003, 57:155 176.

11. Meyer MG, Park S, Zeringue L, Staley M, McKinstry M, Kaufman Rl, Zhang H, Yan D, Yennawar N, Yennawar H, Farber GK, Nixon BT: A dimeric twocomponent receiver domain inhibits the sigma54-dependent ATPase in DctD. FASEB J 2001, 15(7):1326 1328.

12. Leonhartsberger S, Huber A, Lottspeich F, Bock A: The hydH/G Genes from Escherichia coli code for a zinc and lead responsive two-component regulatory system. J Mol Biol 2001, 307(1):93 105.

13. Brahmachary P, Dashti MG, Olson JW, Hoover TR: Helicobacter pylori FlgR is an enhancer-independent activator of sigma54-RNA polymerase holoenzyme. J Bacteriol 2004, 186(14):4535 4542.

14. Hopper S, Bock A: Effector-mediated stimulation of ATPase activity by the sigma 54-dependent transcriptional activator FHLA from Escherichia coli. J Bacteriol 1995, 177(10):2798 2803.

15. Henry JT, Crosson S: Ligand-binding PAS domains in a genomic, cellular, and structural context. Annu Rev Microbiol 2011, 65:261 286.

16. Pittard J, Camakaris H, Yang J: The TyrR regulon. Mol Microbiol 2005, 55(1):16 26.

17. Liu G, Song L, Shu C, Wang P, Deng C, Peng Q, Lereclus D, Wang X, Huang D, Zhang J, Song F: Complete genome sequence of Bacillus thuringiensis subsp. kurstaki strain HD73. Genome Announcements 2013, 1(2):e0008013.

18. Dower WJ, Miller JF, Ragsdale CW: High efficiency transformation of E. coli by high voltage electroporation. Nucleic Acids Res 1988, 16(13):6127 6145

19. Lereclus D, Arantes O, Chaufaux J, Lecadet M: Transformation and expression of a cloned delta-endotoxin gene in Bacillus thuringiensis. FEMS Microbiol Lett 1989, 51(1):211 217.

20. Schaeffer P, Millet J, Aubert JP: Catabolic repression of bacterial sporulation. Proc Natl Acad Sci U S A 1965, 54(3):704 711

21. Du L, Qiu L, Peng Q, Lereclus D, Zhang J, Song F, Huang D: Identification of the promoter in the intergenic region between orf1 and cry8Ea1 controlled by sigma $\mathrm{H}$ factor. App/ Environ Microbiol 2012, 78(12):4164 4168.

22. Li R, Liu G, Xie Z, He X, Chen W, Deng Z, Tan H: PolY, a transcriptional regulator with ATPase activity, directly activates transcription of polR in polyoxin biosynthesis in Streptomyces cacaoi. Mol Microbiol 2010, 75(2):349 364

23. Zianni M, Tessanne K, Merighi M, Laguna R, Tabita FR: Identification of the DNA bases of a DNase I footprint by the use of dye primer sequencing on an automated capillary DNA analysis instrument. J Biomol Tech 2006, 17(2):103 113

24. Agaisse $H$, Lereclus D: Structural and functional analysis of the promoter region involved in full expression of the cryllIA toxin gene of Bacillus thuringiensis. Mol Microbiol 1994, 13(1):97 107

25. Lereclus $D$, Arantes $O: s p b A$ locus ensures the segregational stability of pTH1030, a novel type of gram-positive replicon. Mol Microbiol 1992 6(1):35 46.

26. Bouillaut L, Ramarao N, Buisson C, Gilois N, Gohar M, Lereclus D, Nielsen-Leroux C: FlhA influences Bacillus thuringiensis PlcR-regulated gene transcription, protein production, and virulence. Appl Environ Microbiol 2005, 71(12):8903 8910.

27. Francke C, Groot Kormelink T, Hagemeijer Y, Overmars L, Sluijter V, Moezelaar R, Siezen RJ: Comparative analyses imply that the enigmatic Sigma factor 54 is a central controller of the bacterial exterior. BMC Genomics 2011, 12:385.
28. Barrios H, Valderrama B, Morett E: Compilation and analysis of sigma(54)dependent promoter sequences. Nucleic Acids Res 1999, 27(22):4305 4313.

29. Jiang ZY, Bauer CE: Analysis of a chemotaxis operon from Rhodospirillum centenum. J Bacterio/ 1997, 179(18):5712 5719.

30. Wozniak DJ: Integration host factor and sequences downstream of the Pseudomonas aeruginosa algD transcription start site are required for expression. J Bacteriol 1994, 176(16):5068 5076.

31. Keseler IM, Kaiser D: An early A-signal-dependent gene in Myxococcus xanthus has a sigma 54-like promoter. J Bacteriol 1995, 177(16):4638 4644.

32. Mullin DA, Newton A: A sigma 54 promoter and downstream sequence elements ftr 2 and $f t r 3$ are required for regulated expression of divergent transcription units flaN and flbG in Caulobacter crescentus. J Bacteriol 1993, 175(7):2067 2076.

33. Belitsky BR: Bacillus subtilis GabR, a protein with DNA-binding and aminotransferase domains, is a PLP-dependent transcriptional regulator. J Mol Biol 2004, 340(4):655 664.

34. Edayathumangalam R, Wu R, Garcia R, Wang Y, Wang W, Kreinbring CA, Bach A, Liao J, Stone TA, Terwilliger TC, Hoang QQ, Belitsky BR, Petsko GA, Ringe D, Liu D: Crystal structure of Bacillus subtilis GabR, an autorepressor and transcriptional activator of gabT. Proc Natl Acad Sci U S A 2013, 110(44):17820 17825.

doi:10.1186/s12866-014-0306-3

Cite this article as: Peng et al:: Activation of gab cluster transcription in Bacillus thuringiensis by $\mathrm{\gamma}$-aminobutyric acid or succinic semialdehyde is mediated by the Sigma 54-dependent transcriptional activator GabR. BMC Microbiology 2014 14:306.

\section{Submit your next manuscript to BioMed Central and take full advantage of:}

$\otimes$ Convenient online submission

$\otimes$ Thorough peer review

$\otimes$ No space constraints or color $\nabla$ gure charges

$\nabla$ Immediate publication on acceptance

Q Inclusion in PubMed, CAS, Scopus and Google Scholar

$\otimes$ Research which is freely available for redistribution

Submit your manuscript at www.biomedcentral.com/submit
C Biomed Central 